\title{
PALINDROMIC RHEUMATISM - AN UNUSUAL PRESENTATION OF RHEUMATOID ARTHRITIS
}

\author{
BISWAS $\mathrm{S}^{1}$, RAHMAN A ${ }^{2}$, DAS PP ${ }^{3}, \mathrm{KARIM} \mathrm{ME}^{4}, \mathrm{KHAN} \mathrm{AH}^{5}, \mathrm{AFROSE} \mathrm{R}^{6}, \mathrm{DEY} \mathrm{P}^{7}$

\begin{abstract}
:
We have reported a 24 years old woman presenting with recurrent episodes of joint pain, weakness and fever for last one and half year. Each episode persists for 3/4 days and intervals between episodes are variable. Laboratory investigations showed positive RF and anti-CCP. Each episode remits spontaneously with some NSAIDs. The case high lights the need of accepting Palindromic presentation of early Rheumatoid arthritis and it is not very rare as we think.
\end{abstract}

Key words: Palindrome, Rheumatoid arthritis.

J Dhaka Med Coll. 2013; 22(1) : 93-95.

\section{Introduction:}

Palindromic rheumatism (PR), at first described in 1944, by Hench and Rosenberg as multiple recurring episodes of acute oligoarthritis, periarthritis, and sometimes swelling near the joints and tendons, lasting from a few hours to several days, disappearing completely, and producing no residual articular effects. ${ }^{1}$ It appears that palindromic syndrome is a diverse entity, encompassing other inflammatory conditions at early stages of their evolution, and whose relationship with rheumatoid arthritis (RA) is evident but still unclear. Evolution of up to $50 \%$ of these cases into otherwise typical RA, commonly accompanied by the conversion to rheumatoid factor seropositivity, the frequent occurrence of nodules, the reported response to RA treatment. The prevalence of $P R$ in the population has been estimated at $5 \% ;^{2}$ however, a recent study on the prevalence of $\mathrm{PR}$ as diagnosed by rheumatologists found a prevalence nearly 10 times greater. ${ }^{3}$ Thus, awareness of the typical clinical features and natural history of this condition could aid the primary care physician in its identification and referral to a specialist.

\section{Case report}

A 24 years old woman hailing from Sherpur, presented to Dhaka Medical College Hospital with the complaints of fever for 3 days and pain and weakness both upper and lower limbs for same duration. According to her she was reasonably well $1 \frac{1}{2}$ years back. All on a sudden she developed high fever which was intermittent and associated with chills and rigor. It lasted for 4 days. Along with this fever she felt profound weakness in both upper and lower limbs, that she could not stand from sitting position and could not lift her hand to comb hair. She noticed pain in shoulder \& pelvic girdle and muscles of upper and lower limbs, but the weakness was more profound than pain. When the fever subsided these weakness and pain also went away. She began to move and work normally. After 1 month this whole episode repeated again and she became alright after 3-4 days. Afterwards the gap between these illness episodes became shorter gradually and she began to fall sick frequently. All through this period she has reduced appetite and loses weight. She has no history of oral ulceration, rash or alopecia, no history suggestive of thyroidal illness. Muscle

1. Dr. Sarmistha Biswas, Assistant Professor, Department of Medicine, Dhaka Medical College, Dhaka.

2. Dr. Anonnya Rahman, Indoor Medical Officer, Department of Medicine, Dhaka Medical College Hospital, Dhaka.

3. Dr. Partha Pratim Das, Assistant Professor, Department of Medicine, Dhaka Medical College, Dhaka.

4. Prof. Md. Enamul Karim, Professor of Medicine, Dhaka Medical College, Dhaka.

5. Dr. Abed Hussain Khan, Medical Officer, Bangabandhu Sheikh Mujib Medical University, Dhaka.

6. Dr. Rafia Afrose, Medical Officer, Dhaka Dental College, Dhaka.

7. Dr. Pritam Dey, Honorary Medical Officer, Department of Medicine, Dhaka Medical College Hospital, Dhaka Correspondence: Dr. Sarmistha Biswas, Assistant Professor, Department of Medicine, Dhaka Medical College, Dhaka. 
weakness was not followed by carbohydrate meal. She has no significant past history or family history of note. Her menstrual cycle was normal.

She is mildly anaemic and weighed $41 \mathrm{~kg}$. She has no lymphadenopathy or thyromegaly. There is grade 2 tenderness in small joints of hands, shoulder and elbow joints. Muscle power was $4 / 5$, more diminished proximally than distally. Jerks are normal, all sensory modalities are intact. Other systemic examination reveals no abnormality.

Laboratory investigations revealed WBC 4800/ $\mathrm{mm}^{3}$ with $59 \%$ neutrophils and $34 \%$ lymphocytes. Platelet count was $280000 / \mathrm{mm}^{3}$. ESR was $40 \mathrm{~mm}$ in $1^{\text {st }}$ hour. CRP $6 \mathrm{mg} / \mathrm{dl}$. RA test positive, Serum anti CCP $33.4 \mathrm{U} / \mathrm{ml}$ (positive). Serum electrolytes: $\mathrm{K}^{+} 2.2 \mathrm{mmol} / \mathrm{L}$, $\mathrm{HCO}_{3}{ }^{-} 15 \mathrm{mmol} / \mathrm{L}$. ANA $26.5 \mathrm{U} / \mathrm{ml}$ (positive), Anti ds-DNA negative. Serum CPK 99 U/L (normal 21-232U/L). Serum Uric acid $7.4 \mathrm{mg} /$ dl (normal 2.4-6.0mg/dl). Serum FT $41.16 \mathrm{mg} /$ dl (normal 0.71-1.85mg/dl), Serum TSH 2.58 $\mathrm{IU} / \mathrm{ml}$ (normal 0.47-5.01 IU/ml), $24 \mathrm{hr}$ urine electrolytes: Normal. Renal and liver function tests were normal. X-rays of involved joints were normal.

The patient was treated with NSAIDs and discharged with hydroxychloroquine. She was advised to come for follow up quarterly.

\section{Discussion:}

The incidence of PR is relatively equal in each sex, with a female to male ratio that varies between $1.7: 1$ and $0.55: 1.4$ The mean age of onset of $P R$ is about 45 years, with cases reported from the early 20 s to the $80 \mathrm{~s} .4$ The joints most commonly affected in PR are the shoulders, knees, wrists, ankles, and small joints of the hand. The hips, elbows, and feet are less often involved; however, PR can affect any joint. $\underline{5}$ Although several joints can be affected at the same time, PR is usually monoarticular. The joint involvement is asymmetric and migratory, and patients frequently complain of very severe pain. An attack of PR begins with slowly worsening joint pain that peaks within a few hours and usually subsides within 48 hours. PR attacks may vary in frequency, from one each day to one per month. Asymptomatic periods between attacks are also variable. Redness and swelling of a joint or swelling near a joint is frequently associated with PR. $\underline{6}$ Constitutional symptoms like fever and fatigue may occur during presentation, muscle weakness or tenderness may present during flare. In some cases, transient intracutaneous or subcutaneous nodules on the hands have been described, disappearing within a few days of the attack. ${ }^{5}$

Due to the symptoms of PR and the nature of the attacks, diagnosis can be difficult or take time. The symptoms can be similar to many other forms of arthritis or other auto-immune diseases. It is often a case of eliminating the other conditions before getting the correct diagnosis due to there being no specific test for PR diagnosis. Palindromic rheumatism must be distinguished from acute gouty arthritis and an atypical, acute onset of rheumatoid arthritis (RA). Without specific tests (such as analysis of joint fluid), it may be difficult to distinguish palindromic rheumatism from other episodic joint problems. It is important to note that a person may experience more than one autoimmune disorder at the same time. Laboratory findings are usually normal. Blood tests may show an elevation of the ESR and CRP, but are otherwise unremarkable. Rheumatoid factor may be present especially in the group that is likely to develop Rheumatoid arthritis. Anticitrullinated protein antibody is frequently associated. ${ }^{1}$ It has been suggested that it is an abortive form of rheumatoid arthritis, since anti-cyclic citrullinated peptide antibodies (anti-CCP) and antikeratin antibodies (AKA) are present in a high proportion of patients, as is the case in rheumatoid arthritis. Palindromic rheumatism is frequently the presentation for Whipple disease. Investigators have also found a clinical association between antiphospholipid syndrome and PR. ${ }^{7}$

S. electrolytes points towards the association of hypokaelemia due to renal tubular acidosis in this patient. Renal tubular acidosis (distal or type 1) is also associated with rheumatoid arthritis, hypokaelemia is often severe in this case. ${ }^{8}$ 
White blood cell counts are normal and ANA studies are usually negative. In this patient, the presence of positive ANA suggests the diagnosis of possible SLE. The joint manifestations are typical for PR. Radiographic studies are also always normal in PR. Thus, $\mathrm{PR}$ can be diagnosed by the typical joint involvement, with normal blood tests and normal x-rays, after ruling out other forms of arthritides.

only about one-third of PR cases progress to RA. 9 Factors which predict progression include a positive rheumatoid factor (RF) and small joint involvement early in the disease. $\frac{8}{\text { Additionally, }}$ a positive anti-CCP antibody test within the first year of presentation of PR is highly suggestive for progression to RA. 10 Other chronic diseases that have been seen to develop in patients with PR include systemic lupus erythematosus, seronegative spondyloarthropathies, including psoriatic arthritis and rarely, Wegener granulomatosis. $\underline{6}$ In this patient, both $\mathrm{RF}$ and anti-CCP were positive which portends a likelihood of progression to RA.

Acute attacks of PR may respond favorably to nonsteroidal anti-inflammatory drugs (NSAIDs). Several drugs used for the treatment of RA like antimalarials, gold salts, penicillamine, sulfasalazine, methotrexate, and TNF- á inhibitors have been used in PR. A retrospective study has shown that the use of antimalarials in patients with PR may be associated with a reduction in the risk of developing subsequent RA or other connective tissue disease. 11 Antimalarials are seen to have a role in some patients in preventing attacks or reducing the frequency and intensity of attacks, and hydroxychloroquine was found to be very useful in our patient.

In conclusion, PR should be considered in the differential diagnosis of recurrent attacks of migrating oligoarthritis, presenting with constitutional symptoms when laboratory tests and imaging studies are normal. It is important to identify this condition so that patients may be counseled about the possibility of developing chronic autoimmune conditions, and treated in the appropriate cases.

\section{References:}

1. Hench PS, Rosenberg EF. Palindromic rheumatism. "new" oft-recurring disease of joints (arthritis, periarthritis, para-arthritis) apparently producing no articular residues. Report of thirtyfour cases; its relation to "angio-neural arthrososis," "allergic rheumatism" and rheumatoid arthritis. Arch Intern Med 1944; 73: 293-321.

2. Pasero G, Barbieri P. Palindromic rheumatism: you just have to think about it. Clin Exp Rheumatol 1986; 4: 197-9.

3. Powell A, Davis P, Jones N, et al. Palindromic rheumatism is a common disease: comparison of new-onset palindromic rheumatism compared to new-onset rheumatoid arthritis in a 2-year cohort of patients. J Rheumatol 2008; 35: 992-4.

4. Guerne PA, Weisman MH. Palindromic rheumatism: part of or apart from the spectrum of rheumatoid arthritis. Am J Med 1992; 93: 45160 .

5. Mattingly S. Palindromic rheumatism. Ann Rheum Dis 1966; 25: 307-17.

6. Sanmarti R, Cañete JD, Salvador G. Palindromic rheumatism and other relapsing arthritis. Best Pract Res Clin Rheumatol 2004; 18: 647-61.

7. Panush RS. Food induced (allergic) arthritis: clinical and serologic studies. J Rheumatol 1990; 17: 291-4.

8. Nesher G, Mates M. Palindromic rheumatism: effect of dietary manipulation. Clin Exp Rheumatol 2000; 18: 375-8.

9. Reitblat T, Litinsky I, Caspi D, et al. Antiphospholipid syndrome and palindromic rheumatism: a new possible association. Rheumatol Int 2005; 25: 595-8.

10. Wrong OM, Feest TG, MacIver AG. Immune-related potassium-losing interstitial nephritis: a comparison with distal renal tubular acidosis. Q J Med 1993; 86(8): 513-34.

11. Gonzalez-Lopez L, Gamez-Nava JI, Jhangri GS, et al. Prognostic factors for the development of rheumatoid arthritis and other connective tissue diseases in patients with palindromic rheumatism. J Rheumatol 1999; 26: 540-5. 